\title{
Risk and protective factors affecting sensory recovery after breast reconstruction
}

\author{
Jae Young Bae ${ }^{1}$, Ha Young Shin ${ }^{2}$, Seung Yong Song ${ }^{1}$, Dong Won Lee ${ }^{1}$ \\ ${ }^{1}$ Department of Plastic and Reconstructive Surgery, Institute for Human Tissue Restoration and ${ }^{2}$ Department of Neurology, Yonsei University \\ College of Medicine, Seoul, Korea
}

\begin{abstract}
Background Although loss of sensation in patients with breast cancer after mastectomy followed by breast reconstruction is an important factor affecting patients' quality of life, the mechanism of sensory recovery is still unclear. Our study aimed to identify variables that affect sensory recovery, especially pain, in reconstructed breasts.

Methods All patients with breast cancer who underwent mastectomy followed by immediate breast reconstruction, including nipple reconstruction or areolar tattooing, were included in this study. Sensation was evaluated in the nipple as an endpoint of sensation recovery of the whole breast. Patients rated pain severity using a 3-point verbal rating scale (VRS): grade 0 , no pain; grade 1, mild to moderate pain; and grade 2, severe pain. The VRS was assessed by a single experienced plastic surgeon.

Results In the univariate analysis, the odds ratio (OR) for sensation recovery was 0.951 for age $(P=0.014), 0.803$ for body mass index $(P=0.001), 0.996$ for breast volume before surgery $(P=0.001), 0.998$ for specimen weight after mastectomy $(P=0.040)$, and 1.066 for the period between mastectomy and sensory assessment $(P=0.003)$. In the multivariate analysis, the variables that showed a significant effect were age $(O R, 0.953 ; P=0.034)$, the period between mastectomy and sensory assessment $(O R, 1.071 ; P=0.006)$, and reconstruction using abdominal tissue instead of prosthetic reconstruction $(O R, 0.270 ; P=0.004)$.

Conclusions Based on our results, it can be inferred that aging has a negative impact on the recovery of sensation, breast sensation improves with time after surgery, and the recovery of sensation is better in prosthetic reconstruction.
\end{abstract}

Keywords Mammoplasty / Sensation / Risk factors

\author{
Correspondence: Dong Won Lee \\ Department of Plastic and \\ Reconstructive Surgery, Institute for \\ Human Tissue Restoration, Yonsei \\ University College of Medicine, 50-1 \\ Yonsei-ro, Seodaemun-gu, Seoul \\ 03722 , Korea \\ Tel: +82-2-2228-2215 \\ Fax: +82-2-393-6947 \\ E-mail:xyphoss@yuhs.ac
}

This study was supported by a faculty research grant of Yonsei University College of Medicine (6-2015-0075).

Received: June 24, 2020 • Revised: October 23, 2020 • Accepted: October 27, 2020

pISSN: 2234-6163 • elSSN: 2234-6171 • https://doi.org/10.5999/aps.2020.01151 • Arch Plast Surg 2021;48:26-32

\section{INTRODUCTION}

Surgery is crucial for the treatment of breast cancer, and breast reconstruction is one of the most important procedures that is performed in patients after breast cancer surgery. With improvements in surgical techniques, the goals of breast reconstruction have shifted from simply covering the large defect of the breast after mastectomy to the reconstruction of more realistic and aesthetically pleasing breasts. Reconstruction methods have also evolved through the decades. Advances in surgical equipment and the outcomes of numerous studies have encouraged more sophisticated surgical procedures with fewer complications and a shorter duration. With improvements in surgical outcomes, aesthetic results are also becoming better. However, 
although aesthetically favorable results are the goal for plastic surgeons, patients may not be satisfied with the results if the reconstructed breasts lack sensation. Spear and Ganz [1] stated that restoring sensation in the reconstructed breasts is the next challenge.

Sensations in the breast include pain (nociceptors), touch and pressure (tactile receptors), heat and cold sensation (thermic receptors), itching (pruriceptors), and-as the most complicated factor-erogenous sensations. The sensory nerves supplying the breast mainly comprise the anterolateral branch and anteromedial branch of the intercostal nerve. The anterolateral branches of the third through sixth intercostal nerves reach from the lateral side of the breast to the nipple-areolar complex and provide sensation to the lateral part of the breast, while the anteromedial branches of the second to sixth intercostal nerves run along the internal mammary artery perforators and provide sensation to the medial part of the breast $[2,3]$. These nerves, which are intertwined within the breast, are generally removed along with glandular tissue during mastectomy. For restoration of breast sensation, surgeons are increasingly focusing on re-innervation during breast reconstruction. Many studies have compared innervated and non-innervated breast reconstructions and showed that innervated breast reconstructions were superior for sensory recovery [4-6], while some studies reported that spontaneous re-innervation was also acceptable [7-9].

It has been established that sensation is restored in non-innervated breasts with time. Although several studies have reported factors contributing to the restoration of sensation in reconstructed breasts, the mechanism of sensory restoration is still unclear [10-12]. Previous studies dealing with breast sensation recovery compared two reconstruction methods or compared one method with a control group [13], but no study has compared sensory recovery in terms of pain sensation across three different reconstruction methods at the same time, including a wide range of variables that could possibly affect sensory recovery. Therefore, this study aimed to identify variables that affect sensory recovery, especially pain, in reconstructed breasts and to evaluate which reconstructive option, among non-innervated reconstructions, is most favorable for restoring breast sensation.

\section{METHODS}

\section{Study population}

All patients with breast cancer who underwent mastectomy followed by immediate breast reconstruction, including nipple reconstruction or areolar tattooing, were eligible for inclusion in the study. Patients aged $<18$ years and those who did not require nipple-areolar complex reconstruction or areolar tattooing were excluded from the study. Patients who underwent nipplesparing mastectomy (NSM) were only included if they required nipple-areolar reconstruction or tattooing when a nipple deformity or the nipple was removed due to nipple ischemia after surgery. This study was approved by the Institutional Review Board of Severance Hospital, Yonsei University Health System and informed consent was waived (IRB No. 4-2019-1146).

\section{Demographics}

Patients' age, body mass index (BMI), preoperatively estimated breast volume, mastectomy specimen weight, mastectomy method, reconstruction method, adjuvant therapy (including radiotherapy and chemotherapy), and the period between mastectomy and sensory assessment were recorded. Mastectomy methods were classified into (1) modified radical mastectomy (MRM), (2) total mastectomy (TM), (3) skin-sparing mastectomy (SSM), and (4) NSM. If the axillary lymph nodes were dissected during TM, the procedure was classified as MRM. Prior to surgery, preoperative breast volume was obtained by three-dimensional surface imaging (Axis Three; AX3 Technologies, Miami, FL, USA) [14]. Breast reconstruction methods were classified into (1) prosthetic reconstruction, (2) reconstruction using abdominal tissue, such as a free transverse rectus abdominis musculocutaneous flap or a deep inferior epigastric artery perforator flap, and (3) latissimus dorsi (LD) flap reconstruction. Most of the prosthetic reconstructions were performed using a tissue expander, since the patients had lost their breast skin envelope after mastectomy. The LD group underwent reconstruction using the $\mathrm{LD}$ muscle with or without a breast implant.

\section{Sensory testing}

Pain sensation was assessed in all patients when they visited our clinic for nipple reconstruction or areolar tattooing. At the beginning of each procedure, a local anesthetic agent was injected using a 26-gauge needle, and patients were asked about the intensity of pain that they felt when the skin was pricked with the needle. The needle pricked the site where the new nipple would be located, either based on the location of the nipple on the contralateral breast or where the new nipple was located in the tattooing procedure. The nipple was chosen for the assessment of sensation because several previous studies revealed that the latest place where sensation recovery occurs in the breast is the nipple-areolar complex for all reconstruction methods [15-17]. The assessment point of the patients who underwent prosthesis-based reconstruction was the breast skin flap, while the assessment point of patients who underwent reconstruction with abdominal tissue or an LD flap was the donor skin flap, where 
the nipple was reconstructed. Pain severity was rated by the patients using a 3-point verbal rating scale (VRS): grade 0 , no pain; grade 1 , mild to moderate pain; and grade 2 , severe pain. It is known that VRS has good validity and reliability as a measure of pain intensity in many languages [18]. The VRS was assessed by a single experienced plastic surgeon (DWL).

\section{Statistical analysis}

All data were compiled in Microsoft Excel (Microsoft Corp., Redmond, WA, USA). Means and standard deviations were used to summarize the continuous variables. Frequencies and proportions were used to present the categorical characteristics. Univariate and multivariate analysis was performed using an ordinal logistic regression model to assess the predictors of risk and protective factors for sensory recovery. As the value of variables increased, the odds of a higher pain score were shown by the odds ratio, which indicates better sensory recovery. Multivariate analysis was performed for factors that were found to have a significant effect on the outcome variable in the univariate analysis. Odds ratios were calculated for implant-based reconstruction and reconstruction using abdominal tissue. An analysis using $\mathrm{LD}$ reconstruction as the reference was not performed because the odds ratio has a reciprocal relationship and the P-value is the same as in the calculations based on implantbased reconstruction and reconstruction using abdominal tissue. The threshold for statistical significance was set at $\mathrm{P}<0.05$. However, after adjusting the P-values for multiple comparisons, factors with adjusted P-values below 0.0167 were considered significant for the reconstruction and mastectomy method. All analyses were performed using SAS 9.4 (SAS Institute, Inc., Cary, NC, USA).

\section{RESULTS}

In total, 127 patients were included in the study. Patients' demographic characteristics are presented in Table 1. The average age of the patients was $50.8 \pm 8.3$ years, and their average BMI was $22.8 \pm 2.8 \mathrm{~kg} / \mathrm{m}^{2}$. The average estimated breast volume prior to surgery and the specimen weight after surgery was $330.0 \pm 147.2$ $\mathrm{mL}$ and $434.0 \pm 212.8 \mathrm{~g}$, respectively. TM was performed in 85 patients (66.9\%), MRM in 10 patients (7.9\%), SSM in 20 patients (15.8\%), and NSM in 12 patients (9.4\%). Sixteen patients (12.6\%) received radiotherapy, and 44 patients $(34.6 \%)$ underwent chemotherapy; neoadjuvant chemotherapy was administered in nine patients $(7.1 \%)$, postoperative chemotherapy in 26 patients $(20.5 \%)$, and nine patients $(7.1 \%)$ received both neoadjuvant and adjuvant chemotherapy. Prosthetic reconstruction was performed in 57 patients (44.9\%), reconstruction using the
Table 1. Patient demographics

\begin{tabular}{lc}
\hline Variable & Value $(\mathrm{n}=127)$ \\
\hline Age $(\mathrm{yr})$ & $50.8 \pm 8.3(31-75)$ \\
Body mass index $\left(\mathrm{kg} / \mathrm{m}^{2}\right)$ & $22.8 \pm 2.8(17.75-35.35)$ \\
Preoperatively estimated breast volume (mL) & $330.0 \pm 147.2(64-895)$ \\
Mastectomy specimen weight (g) & $434.0 \pm 212.8(64-1,476)$ \\
Period between mastectomy and sensory & $13.0 \pm 8.1(3-45)$ \\
$\quad$ & \\
assessment (mon) & \\
Mastectomy method & $85(66.9)$ \\
TM & $10(7.9)$ \\
SRM & $20(15.8)$ \\
$\quad$ NSM & $12(9.4)$ \\
Radiotherapy & $16(12.6)$ \\
Chemotherapy & $44(34.6)$ \\
Reconstruction method & \\
Implant-based reconstruction & $57(44.9)$ \\
Reconstruction using abdominal tissue & $41(32.3)$ \\
LD reconstruction & $29(22.8)$ \\
\hline
\end{tabular}

Values are presented as mean \pm SD (range) or number (\%).

TM, total mastectomy; MRM, modified radical mastectomy; SSM, skin-sparing mastectomy; NSM, nipple-sparing mastectomy; LD, latissimus dorsi.

abdominal tissues was performed in 41 patients (32.3\%), and the LD flap was used for reconstruction in 29 patients (22.8\%). The mean period between mastectomy and sensory assessment was $13.0 \pm 8.13$ months.

The odds ratio for each variable in the univariate analysis is shown in Table 2. The odds ratio for patients' age was 0.951 (95\% confidence interval [CI], 0.912-0.990; $\mathrm{P}=0.014$ ), which suggests that the likelihood of developing pain sensation decreased with age. The odds ratios for BMI, estimated breast volume, specimen weight, and the period between mastectomy and sensory assessment showed statistical significance, while no significance was found for radiotherapy, chemotherapy, and mastectomy method. In the analysis of the reconstruction method, with prosthetic reconstruction used as the reference, the odds ratio for reconstruction using abdominal tissue was significant (0.189; 95\% CI, 0.084-0.426; P $<0.001)$, while the odds ratio for LD flap reconstruction was 0.413 (95\% CI, 0.175$0.973 ; \mathrm{P}=0.043$ ). There was no significant difference between $\mathrm{LD}$ and other reconstruction methods.

Ordinal logistic regression was performed for multivariate analysis for all relevant covariates that showed a significant odds ratio in the univariate analysis, which were patients' age, BMI, estimated breast volume, mastectomy specimen weight, mastectomy method, reconstruction method, and the period between mastectomy and sensory assessment. Statistical significance was observed for age, reconstruction method, and the period between mastectomy and sensory assessment: the odds ratio for age was 0.953 (95\% CI, 0.912-0.996; $\mathrm{P}=0.034$ ). The odds ra- 
Table 2. Univariate analysis of factors that may affect patients' pain sensation using ordinal logistic regression

\begin{tabular}{|c|c|c|}
\hline Variable & Odds ratio $(95 \% \mathrm{Cl})$ & P-value \\
\hline Age (yr) & $0.951(0.912-0.990)$ & 0.014 \\
\hline Body mass index $\left(\mathrm{kg} / \mathrm{m}^{2}\right)$ & $0.803(0.706-0.914)$ & 0.001 \\
\hline Preoperatively estimated breast volume (mL) & $0.996(0.994-0.998)$ & 0.001 \\
\hline Mastectomy specimen weight (g) & $0.998(0.997-1.000)$ & 0.040 \\
\hline Period between mastectomy and sensory assessment (mon) & $1.066(1.021-1.114)$ & 0.003 \\
\hline \multicolumn{3}{|l|}{ Mastectomy method } \\
\hline MRM vs. TMa) & $1.206(0.355-4.098)$ & 0.763 \\
\hline SSM vs. TMa) & $0.409(0.161-1.035)$ & 0.059 \\
\hline NSM vs. TMa) & $1.206(0.391-3.731)$ & 0.744 \\
\hline Radiotherapy & $0.885(0.334-2.347)$ & 0.806 \\
\hline Chemotherapy & $1.383(0.699-2.732)$ & 0.352 \\
\hline \multicolumn{3}{|l|}{ Reconstruction method ${ }^{b)}$} \\
\hline Reconstruction using abdominal tissue vs. implanta) & $0.189(0.084-0.426)$ & $<0.001$ \\
\hline LD reconstruction vs. implanta) & $0.413(0.175-0.973)$ & 0.043 \\
\hline LD reconstruction vs. reconstruction using abdominal tissue $e^{a)}$ & $2.179(0.878-5.405)$ & 0.093 \\
\hline
\end{tabular}

An odds ratio greater than 1 implies a higher likelihood of experiencing pain.

$\mathrm{Cl}$, confidence interval; TM, total mastectomy; MRM, modified radical mastectomy; SSM, skin-sparing mastectomy; NSM, nipple-sparing mastectomy; LD, latissimus dorsi. ${ }^{a}$ Reference. The odds ratio has a reciprocal relationship when the reference variable is switched to another; ${ }^{\text {b) }}$ Ordinal logistic regression was performed using implant reconstruction and reconstruction using abdominal tissue as a reference. Due to multiple comparisons, it is reasonable to view the significance level as $0.05 / 3=0.0167$ for variables.

Table 3. Multivariate analysis of all relevant univariate factors affecting patients' pain sensation that showed significant odds ratios using ordinal logistic regression

\begin{tabular}{lcc}
\hline Variable & Odds ratio $(95 \% \mathrm{Cl})$ & P-value \\
\hline Age $(\mathrm{yr})$ & $0.953(0.912-0.996)$ & 0.034 \\
Body mass index $\left(\mathrm{kg} / \mathrm{m}^{2}\right)$ & $0.898(0.765-1.056)$ & 0.193 \\
Preoperatively estimated breast volume $(\mathrm{mL})$ & $0.996(0.993-1.001)$ & 0.058 \\
Mastectomy specimen weight (g) & $1.000(0.998-1.003)$ & 0.798 \\
Period between mastectomy and sensory assessment (mon) & $1.071(1.019-1.124)$ & 0.006 \\
Reconstruction method) & & 0.004 \\
Reconstruction using abdominal tissue vs. implanta) & $0.270(0.110-0.664)$ & 0.205 \\
LD reconstruction vs. implanta) & $0.542(0.210-1.397)$ & 0.167 \\
LD reconstruction vs. reconstruction using abdominal tissue & $2.004(0.748-5.376)$ & \\
\hline
\end{tabular}

An odds ratio greater than 1 implies a higher likelihood of experiencing pain.

$\mathrm{Cl}$, confidence interval; LD, latissimus dorsi.

${ }^{a}$ Reference. The odds ratio has a reciprocal relationship when the reference variable is switched to another; ${ }^{b}$ Ordinal logistic regression was performed using implant reconstruction and reconstruction using abdominal tissue as a reference. Due to multiple comparisons, it is reasonable to view the significance level as $0.05 / 3=0.0167$ for variables.

tios for the period between mastectomy and sensory assessment, and for reconstruction using abdominal tissue instead of prosthetic reconstruction were 1.071 (95\% CI, 1.019-1.124; $\mathrm{P}=0.006)$ and 0.270 (95\% CI, 0.110-0.664; $\mathrm{P}=0.004)$, respectively. The odds ratio for prosthetic reconstruction with reconstruction using abdominal tissue as the reference group was 3.698 (95\% CI, 1.506-9.081; $\mathrm{P}=0.004$ ), which is reciprocal to the odds ratio for reconstruction using abdominal tissue with prosthetic reconstruction as the reference group (Table 3 ). These results indicate that pain sensation decreased with age and increased as the period between mastectomy and sensory assessment became longer. Prosthetic reconstruction achieved better sensation than reconstruction using abdominal tissue, while $\mathrm{LD}$ reconstruction showed no significant difference from the other two reconstruction methods.

\section{DISCUSSION}

This study found that breast sensation improved with time after surgery, and prosthetic reconstruction showed better results in restoring breast sensation than reconstruction with abdominal tissue. Sensory recovery due to nerve regeneration is an important determinant of the outcomes of breast reconstruction surgery. Following nerve injury, sensory recovery may occur 
through axonal regeneration or re-innervation from neighboring nerves [19]. It takes a long time for nerve recovery to occur after mastectomy. Previous studies have shown that improved sensation has a positive impact on patient-rated quality of life $[6,20]$. Therefore, it is important to educate patients before surgery regarding the expected progress of breast sensation recovery. To this end, it is necessary to periodically measure sensory recovery in patients who have undergone surgery and to estimate the time required for recovery.

Many studies have been conducted regarding the recovery of breast sensation after reconstruction. According to Shridharani et al. [13], who conducted a systematic review of published articles dealing with breast sensation after breast reconstruction, reconstruction with abdominal tissue led to the most favorable recovery of sensation, followed by $\mathrm{LD}$ reconstruction and finally implants, although this could not be statistically quantified through a meta-analysis. Our study concords with the findings of Khan et al. [21], who reported better pleasurable sensation in patients who underwent prosthetic reconstruction than in those who underwent autologous reconstruction, and also found that radiotherapy or chemotherapy did not affect sensation. Beugels et al. [16] also reported a similar result to the findings of our study that sensory recovery was significantly associated with length of follow-up.

Based on the univariate model, our results suggest that as age, $\mathrm{BMI}$, breast volume before surgery, and weight of the mastectomy specimen increased, the pain score decreased. However, more pain sensation was felt in patients as the period between mastectomy and sensory assessment became longer. A plausible explanation for the findings of the univariate model (namely, that a high BMI, large breast volume, and heavy mastectomy specimen disturbed sensory recovery) is that patients with a higher BMI-which may have a positive correlation with larger breast volume, and therefore a heavier mastectomy specimenmay require nerves to grow in a larger area for sensation to recover. In the multivariate model, older patients felt less pain, and a stronger pain sensation was felt as the period between mastectomy and sensory assessment became longer. Patients who underwent prosthetic reconstruction felt more pain sensation than those who underwent reconstruction using abdominal tissue, in both the univariate and multivariate analyses. No significant differences were found between the LD group and other groups. A possible mechanistic explanation for the finding of better sensation in the prosthetic group than in the autologous group is as follows. First, nerves in the breast skin may grow more quickly than those passing through discontinuous autologous soft tissue or skin, leading to more favorable findings in the prosthetic group when sensation in the breast skin was assessed. Second, intact nerves passing through the subdermal area after mastectomy could be stretched by using a tissue expander in the prosthetic group. A history of receiving chemotherapy and radiotherapy had no effect on sensory recovery in both univariate and multivariate analyses, which is also in accordance with previous studies $[15,16]$.

The limitations of this study include the fact that our study measured pain only at one time point. Compared to other studies, our measurement methods were not as diverse and the objectivity was low because the outcome reflected only the patient's subjective response. In addition, sensory restoration was compared between transferred tissue in the autologous group and the inherent mastectomy flap in the prosthetic group in this study, which may have led to biased results. Although the mechanism underlying sensory recovery in these two groups might be disparate, there is no debate that a patient's perception of sensory stimuli is of paramount importance when discussing clinical implications of sensory recovery. Another limitation is that an evaluation of sensation only at the nipple may not be generalizable to sensation of the whole breast. However, previous studies have proven that the nipple-areolar complex has the worst and most delayed sensation recovery after reconstruction for all three reconstruction methods. Bijkerk et al. [15] reported that the nipple had the lowest sensation recovery of nine assessment points in prosthesis reconstruction. Delay et al. [17] found that the nipple had the lowest sensation recovery of seven assessment points in $\mathrm{LD}$ reconstruction. Beugels et al. [16] also reported that the nipple-areolar complex had the lowest sensation recovery in non-innervated abdominal flaps. Based on these studies, we used the nipple as an endpoint for sensation recovery of the whole breast regardless of the reconstruction method.

Nevertheless, the large sample size is a strength of this study. Additionally, few studies have evaluated pain sensation, whereas almost every study on this topic evaluated touch sensation; however, touch and pain sensation involve different conduction pathways. Furthermore, to date, studies dealing with breast sensation recovery have compared only two reconstruction methods or have compared one method with a control group; to our knowledge, no previous study has compared sensory recovery regarding pain sensation with three different reconstruction methods at the same time including a broad range of variables. Thus, we conducted this study to determine which reconstructive option, among non-innervated reconstruction procedures, is most favorable for restoring breast sensation and to clarify other risk and protective factors affecting sensory recovery after breast reconstruction. To reinforce the findings of our study, further research should be conducted, including examinations 
of multiple points using diverse evaluation methods with longterm follow-up.

Restoration of breast sensation after mastectomy and breast reconstruction is essential for patients' quality of life. Hence, it is important to understand the factors that affect sensory recovery in the breast. Our results suggest that older age has a negative impact on the recovery of breast sensation, and the recovery of breast sensation improves with time after surgery. Prosthetic reconstruction showed better results in terms of recovery of breast sensation than reconstruction using abdominal tissue.

\section{NOTES}

\section{Conflict of interest}

No potential conflict of interest relevant to this article was reported.

\section{Ethical approval}

The study was approved by the Institutional Review Board of Severance Hospital (IRB No. 4-2019-1146) and performed in accordance with the principles of the Declaration of Helsinki.

\section{Author contribution}

Conceptualization: HY Shin, SY Song. Data curation: JY Bae. Funding acquisition: DW Lee. Writing - original draft: JY Bae, HY Shin, SY Song, DW Lee. Writing - review \& editing: JY Bae, HY Shin, SY Song, DW Lee.

\section{ORCID}

Jae Young Bae

Ha Young Shin

Seung Yong Song

Dong Won Lee

https://orcid.org/0000-0002-0258-0606 https://orcid.org/0000-0002-4408-8265 https://orcid.org/0000-0002-3145-7463 https://orcid.org/0000-0003-0046-3139

\section{REFERENCES}

1. Spear SL, Ganz JC. Immediate postmastectomy reconstruction: TRAM flap transposition techniques. In: Methes SJ, Hentz VR, editors. Plastic surgery: Volume 4: Trunk and lower extremity. 2nd ed. Philadelphia: Saunders; 2006. p. 835-47.

2. Beugels J, Cornelissen AJ, Spiegel AJ, et al. Sensory recovery of the breast after innervated and non-innervated autologous breast reconstructions: a systematic review. J Plast Reconstr Aesthet Surg 2017;70:1229-41.

3. Schlenz I, Kuzbari R, Gruber H, et al. The sensitivity of the nipple-areola complex: an anatomic study. Plast Reconstr Surg 2000;105:905-9.
4. Blondeel PN, Demuynck M, Mete D, et al. Sensory nerve repair in perforator flaps for autologous breast reconstruction: sensational or senseless? Br J Plast Surg 1999;52:3744.

5. Spiegel AJ, Menn ZK, Eldor L, et al. Breast reinnervation: DIEP neurotization using the third anterior intercostal nerve. Plast Reconstr Surg Glob Open 2013;1:e72.

6. Temple CL, Tse R, Bettger-Hahn M, et al. Sensibility following innervated free TRAM flap for breast reconstruction. Plast Reconstr Surg 2006;117:2119-27.

7. Santanelli F, Longo B, Angelini M, et al. Prospective computerized analyses of sensibility in breast reconstruction with non-reinnervated DIEP flap. Plast Reconstr Surg 2011; 127:1790-5.

8. Stromps JP, Bozkurt A, Grieb G, et al. Spontaneous reinnervation of deep inferior epigastric perforator flaps after delayed breast reconstruction. J Reconstr Microsurg 2016;32: 169-77.

9. Tindholdt TT, Tonseth KA. Spontaneous reinnervation of deep inferior epigastric artery perforator flaps after secondary breast reconstruction. Scand J Plast Reconstr Surg Hand Surg 2008;42:28-31.

10. Magarakis M, Venkat R, Dellon AL, et al. Pilot study of breast sensation after breast reconstruction: evaluating the effects of radiation therapy and perforator flap neurotization on sensory recovery. Microsurgery 2013;33:421-31.

11. Benediktsson KP, Perbeck L, Geigant E, et al. Touch sensibility in the breast after subcutaneous mastectomy and immediate reconstruction with a prosthesis. Br J Plast Surg 1997;50:443-9.

12. Yano K, Hosokawa K, Takagi S, et al. Breast reconstruction using the sensate latissimus dorsi musculocutaneous flap. Plast Reconstr Surg 2002;109:1897-902.

13. Shridharani SM, Magarakis M, Stapleton SM, et al. Breast sensation after breast reconstruction: a systematic review. J Reconstr Microsurg 2010;26:303-10.

14. Lee WY, Kim MJ, Lew DH, et al. Three-dimensional surface imaging is an effective tool for measuring breast volume: a validation study. Arch Plast Surg 2016;43:430-7.

15. Bijkerk E, van Kuijk SMJ, Beugels J, et al. Breast sensibility after mastectomy and implant-based breast reconstruction. Breast Cancer Res Treat 2019;175:369-78.

16. Beugels J, Cornelissen AJ, van Kuijk SM, et al. Sensory recovery of the breast following innervated and noninnervated DIEP flap breast reconstruction. Plast Reconstr Surg 2019; 144:178e-188e.

17. Delay E, Jorquera F, Lucas R, et al. Sensitivity of breasts reconstructed with the autologous latissimus dorsi flap. Plast 
Reconstr Surg 2000;106:302-9.

18. Jensen MP, Castarlenas E, Roy R, et al. The utility and construct validity of four measures of pain intensity: results from a university-based study in Spain. Pain Med 2019;20: 2411-20.

19. Mofid MM, Dellon AL, Elias JJ, et al. Quantitation of breast sensibility following reduction mammaplasty: a comparison of inferior and medial pedicle techniques. Plast Reconstr
Surg 2002; 109:2283-8.

20. Cornelissen AJ, Beugels J, van Kuijk SM, et al. Sensation of the autologous reconstructed breast improves quality of life: a pilot study. Breast Cancer Res Treat 2018;167:687-95.

21. Khan A, Zhang J, Sollazzo V, et al. Sensory change of the reconstructed breast envelope after skin-sparing mastectomy. Eur J Surg Oncol 2016;42:973-9. 